\title{
Verweildauer kann und sollte weiter sinken
}

\author{
The Lenght of In-Patient-Treatment in Psychiatry Should Further be Decreased, \\ a Necessity Due to New Developments in Treatment
}

Bibliografie

Dol 10.1055/s-2007-986347

Psychiat Prax 2008; 35 :

267-268

(c) Georg Thieme Verlag KG

Stuttgart · New York .

ISSN 0303-4259

Korrespondenzadressen

Dr. med. Wiebke Martinsohn-

\section{Schittkowski}

Kompetenz-Centrum für Psy-

chiatrie und Psychotherapie der medizinischen Dienste und der Spitzenverbände der GKV

Blücherstraße 27c

18055 Rostock

w.martinsohn-schittkowski@

mdk-mv.de

\section{Psychische Erkrankungen - eine Herausforderung an die Gesellschaft $\nabla$}

Einer Studie der Universität Dresden zufolge erkranken jährlich rund ein Viertel aller EU-Bürger an psychischen Störungen [1]. Um dem gerecht zu werden, werden auch in Deutschland Modelle für die Versorgung psychisch kranker Menschen mit komplexen Behandlungsbedürfnissen entwickelt. Die Bedürfnisse von Betroffenen, die Ergebnisse der Versorgungsforschung und politische Regelungen bilden Ausgangspunkte für die weitere Modernisierung in der Psychiatrie. Insbesondere unter der Frage, innerhalb welcher Versorgungsstrukturen psychisch Kranke optimal zu therapieren sind, ist in den letzten Jahren eine Schwerpunktverlagerung weg von den stationären über teilstationäre hin zu den ambulanten Angeboten zu beobachten.

\section{Forderungen Betroffener an eine moderne psychiatrische Behandlung $\nabla$}

Betroffene sehen sich zunehmend für ihre psychische Entwicklung selbst verantwortlich und möchten, dass ihre Krankheitserfahrungen in der Behandlung beachtet, genutzt und umgesetzt werden. Angehörige fordern Informationen und Einbeziehung, um Betroffene in ihrem sozialen Umfeld unterstützen und begleiten zu können. Ziel einer psychiatrischen Behandlung muss es sein, Kranke und ihre Angehörigen zu Fachleuten für ihre Erkrankung und deren Bewältigung zu machen. Behandlung sollte dort angeboten werden, wo ihre Ergebnisse langfristig genutzt werden können - gemeindenah. Sie muss sich am individuellen Bedarf des Erkrankten orientieren und personenzentriert angelegt sein [2].

\section{Wissenschaftliche Begleitforschung \\ $\nabla$}

International ist seit Jahrzehnten ein Wandel hin zu einer gemeindenahen Versorgung selbst akut psychisch Kranker zu beobachten. Diese Entwicklung wurde mit großem wissenschaftlichen Interesse verfolgt.

Aktuelle Erkenntnisse belegen eine der vollstationären Behandlung vergleichbare therapeutische Effektivität der tagesklinischen Behandlung [3]. Die behandelten Diagnosegruppen ähneln denen der stationären Therapie. Tagesklinische Behandlung kommt bei ca. 30\% der bislang stationär behandelten Patienten in Betracht [4]. Die Behandlungsdauer ist nicht notwendigerweise länger als im stationären Bereich [5]. Die tagesklinisch erzielten Behandlungsresultate dürfen heute im Vergleich zur stationären Therapie als zeitstabiler angesehen werden. Offensichtlich gelingt ein Transfer der Therapieergebnisse in die individuellen Lebenswelten durch die Alltagsnähe besser. Akut tagesklinische Therapie ist bei vergleichbarer klinischer Effektivität kostengünstiger als die vollstationäre Behandlung [6]. Auch wurde wiederholt gezeigt, dass psychisch kranke Menschen mit schweren Störungen und wiederholten bzw. lang andauernden Krankenhausaufenthalten deutlich weniger häufig stationäre Krankenhausbehandlung benötigen, wenn sie kontinuierlich ambulant, notfalls auch aufsuchend, behandelt werden [2].

\section{Politische Rahmenbedingungen $\nabla$}

Bundesregierung und Bundesgesetzgeber haben im GKV-Gesundheitsreformgesetz 2000 einige Regelungen getroffen, die als Grundlage der Weiterentwicklung der psychiatrischen Krankenversorgung dienen. Neben der Stärkung der Psychiatrie-Personalverordnung wurden Maßnahmen beschlossen, die eine Ambulantisierung der psy- 
chiatrisch-psychotherapeutischen Behandlung befördern. Seither konnten sich Soziotherapie, ambulante psychiatrische Krankenpflege sowie psychiatrische Institutsambulanzen (PIA) als Kassenleistung etablieren.

In den meisten Bundesländern gibt es spezielle Landesgesetze, in die zunehmend auch Regelungen zur Kooperation und Koordination psychiatrischer Dienste sowie die Schaffung gemeindenaher Versorgungsstrukturen aufgenommen werden.

\section{Wo steht die Psychiatrie aktuell?}

Seit der Psychiatrie-Enquete 1975 haben sich ambulante und teilstationäre Behandlungsangebote immer mehr verbreitet. Heute verfügt beinahe jedes psychiatrische Krankenhaus über eine Tagesklinik und zunehmend auch über eine PIA.

\section{Wandel im Selbstverständnis der Kliniken}

Für psychiatrische Kliniken findet ein Wandel in deren Selbstverständnis statt. Traditionell der Dreh- und Angelpunkt der Behandlung nehmen sie zunehmend eine initiierende Rolle im Sinne einer zusammenarbeitenden Versorgungslandschaft ein. Modellvorhaben zur integrierten Versorgung mit stationären, teilstationären und ambulanten Angeboten unter einem Dach werden durchgeführt. Fallmanager haben häufig ihren Sitz in einer Klinik. Stationäre Behandlungen sind Patienten vorbehalten, die einer Entlastung und einer Herausnahme aus dem sozialen Milieu bedürfen. Hierzu gehören Kriseninterventionen bei schweren Dekompensationen, unfreiwillige Behandlungen aber auch die Behandlung komplexer und therapeutisch schwer beeinflussbarer Krankheiten. Größere Einrichtungen bieten bessere Möglichkeiten zu spezialisierten Therapien für bestimmte Diagnosegruppen $[5,7]$.

\section{Tageskliniken verändern ihr Spektrum}

Tageskliniken führen verstärkt Akutbehandlungen als Alternative zur vollstationären Aufnahme durch. Die tagesklinische Behandlung ist geeignet, wenn die psychische Störung vor allem zu sozialen Defiziten geführt hat und ein besseres Funktionsniveau des Patienten angestrebt wird. Auch Patienten, die neben der Behandlung Angehörige zu versorgen haben, profitieren von diesem Setting [5]. Für einige ausgesuchte Indikationsgebiete erweist sich die Behandlung im tagesklinischen Rahmen sogar als besonders wirksam $[3,8,9]$.

\section{Ambulante Behandlung als Regelbehandlung}

Ambulante Behandlung sollte zunehmend die Regelbehandlung sein. Jede Störung, die nicht so stark ist, dass sie das Leben des Patienten wesentlich behindert, kann ambulant behandelt werden $[8,9]$. Über die Behandlung bei niedergelassenen Nervenärzten hinaus halten viele Kliniken für sogenannte „nicht wartezimmerfähige“ Patienten ein ambulantes Angebot vor (PIA). Als erfolgreich in der Behandlung akuter Krankheitsepisoden für verschiedene Zielgruppen erwies sich in zahlreichen Studien die aufsuchende 24-Stunden-Betreuung als eine Form der gemeindeintegrierten Versorgung.

\section{Vernetzung der medizinischen Leistungen}

Aktuell durchgeführte Modellprojekte widmen sich vorrangig dem Anliegen, vorhandene medizinische Leistungen zu vernetzen und somit therapeutische Brüche zwischen den Behandlungsphasen abzubauen. Überwiegend zielen die Projekte darauf $a b$, sich den Bedürfnissen der Patienten besser anzupassen. Bewährt haben sich personenzentrierte Ansätze, Case-Management-Ansätze und auch das „Home treatment“ [2,9]. Die koordinierende Bezugsperson soll eine gezielte und bedarfsgerechte Nutzung des Gesamtleistungsprofils sicherstellen. In unterschiedlichem Umfang werden stationäre und ambulante medizinische Einrichtungen, Niedergelassene sowie rehabilitative und komplementäre Angebote integriert. Die Koordination der Behandlungsebenen ist auch unter ökonomischen Gesichtspunkten sinnvoll. Beispielsweise geben regionale Budgets/Komplexpauschalen Anreize, bei möglichst geringem Ressourcenverbrauch eine langfristige Beschwerdefreiheit zu erreichen [10].

\section{Wohin führt der Weg? \\ $\nabla$}

Zusammenfassend stellt sich nicht die Frage, $o b$ Verweildauern sinken können und sollten, sondern eher wie der optimale Weg dahin aussieht. Die stattfindende Ambulantisierung der psychiatrischen Behandlung liegt bis auf einige Krankheitsbilder im Interesse der erkrankten Menschen und deren Angehörigen und ist, soweit wissenschaftlich untersucht, als effektiv und ökonomisch zu bezeichnen. $\mathrm{Zu}$ fordern ist der weitere Ausbau einer vertraglich vereinbarten Zusammenarbeit aller Leistungserbringer, um bestehende Unter-, Über- und Fehlversorgungen abzubauen.

\section{Literatur}

1 Wittchen $\mathrm{H}-\mathrm{U}$. Psychische Störungen in Deutschland und der EU Größenordnung und Belastung, 1. Deutscher Präventionskongress, 1. Dezember 2005

2 Schmidt-Zadel R, Kunze H. Mit oder ohne Bett: Personenzentrierte Krankenhausbehandlung im Gemeindepsychiatrischen Verbund, Aktion psychisch Kranke. Bonn: Psychiatrie-Verlag, 2002

3 Marshall $M$ et al. Systematic reviews of the effectiveness of day care with severe mental disorders: (1) acute day hospital versus admission; (2) vocational rehabilitation; (3) day hospital versus outpatient care. Health Technol Assess 2001; 5: 1 - 75

4 Kallert TW et al. Akutpsychiatrische tagesklinische Behandlung: Ein effektivitätsgesichertes Versorgungsangebot? Psychiat Prax 2004; 31: $409-419$

5 Wietersheim J von et al. Status, Möglichkeiten und Grenzen der Behandlung in psychosomatischen Tageskliniken. Psychother Psych Med 2005; 55: 79-83

6 Kallert TW et al. Direkte Kosten akutpsychiatrischer tagesklinischer Behandlung: Ergebnisse aus einer randomisierten kontrollierten Studie. Psychiat Prax 2005; 32: 132 - 141

7 Binswanger M. Ambulant vor teilstationär vor stationär? Thesen und Modelle moderner psychiatrischer Klinikbehandlung. Moderne evidenz-basierte Psychiatrie: Was ist belegt? Jahreskongress der Schweizerischen Gesellschaft für Psychiatrie und Psychotherapie, 2. September 2005

8 LWV Landeswohlfahrtsverband Hessen. Konzept Klinik Bamberger Hof. Arbeitspapier

9 Brenner HD et al. Gemeindeintegrierte Akutversorgung - Möglichkeiten und Grenzen. Nervenarzt 2000; 71: 691 -699

10 Deister $A$ et al. Psychiatrische Versorgung - Kein Anreiz zur Fallzahlausweitung. Deutsches Ärzteblatt 2005; 10: 444-445 